\title{
Controlled mean-field theory for disordered electronic systems: Single-particle properties
}

\author{
R. Vlaming and D. Vollhardt \\ Institut für Theoretische Physik C, Technische Hochschule Aachen, D-5100 Aachen, Federal Republic of Germany
}

(Received 20 May 1991)

\begin{abstract}
A self-consistent, conserving mean-field theory for one-particle properties of disordered electronic systems is presented. It is based on a systematic perturbation expansion in $1 / Z$, where $Z$ is the coordination number of the lattice. To obtain a nontrivial limit for large $Z$, it is crucial to rescale the hopping integral $t$ according to $t \sim 1 / \sqrt{Z}$. In the limit $Z \rightarrow \infty$, the well-known coherent-potential approximation (CPA) is found to become exact for any lattice. Explicit proofs are presented within the locator and propagator formalism. This explains why CPA often yields quantitatively correct results even for values of the disorder not accessible by conventional perturbation theory. Exact results are presented for the Bethe lattice, with the disorder given by a box and a binary-alloy distribution, respectively. Explicit $1 / Z$ corrections to the results for $Z=\infty$ are calculated and the additional effects are discussed.
\end{abstract}

\section{INTRODUCTION}

Investigations involving statistical disorder are usually faced with substantial technical difficulties, particularly when it comes to working in a parameter regime that is not accessible to perturbation theory. In general, even one-particle quantities, such as the density of states (DOS), cannot be calculated exactly. Counterexamples, where exact results have been obtained, are the Lloyd model, ${ }^{1}$ with its Lorentzian probability distribution for the disorder, Wegner's calculation ${ }^{2}$ of the DOS at the band center in the quantum Hall regime, and onedimensional disordered systems. ${ }^{3}$ Nevertheless, in the overwhelming number of cases, one is forced to employ approximation schemes of various kinds. ${ }^{4,5}$ Of these, the coherent-potential approximation ${ }^{6,7}$ (CPA) is probably the most widely used when it comes to calculating oneparticle properties. $^{8-10,4,5}$ This self-consistent approximation is recognized as the best single-site approximation for the spectral properties of disordered systems; indeed, it has the structure of a mean-field approximation. The CPA becomes exact in certain limiting cases (low and high concentrations of impurities, small scattering strengths, atomic limit) and yields an analytic interpolation in between these limits, the validity of which cannot be assessed within the CPA itself. On the other hand, the interpolation in the intermediate regime of parameters is known to yield good quantitative information about systems in dimensions $d=3$ (and even lower), if cluster effects are unimportant. Hence, the CPA is even better than one might expect from the assumptions entering this approximation. It remains to clarify the origin of this success.

Mean-field approximations are widely used to gain insight into gross, overall features of a problem, when an exact solution is not available. To assess the validity of a mean-field theory requires knowledge of a limit in which this theory becomes exact; only then can the theory be improved - at least, in principle - by a perturbation expansion around this limit. It is well known that for classical and quantum spin models (e.g., Ising, Heisenberg) the mean-field results for the free energies become exact for high coordination number $Z$, i.e., for spatial dimension $d \rightarrow \infty .{ }^{11,12}$ However, the situation is quite different in the case of fermionic models, i.e., when there are itinerant quantum-mechanical degrees of freedom. For this class of problems the limit $d \rightarrow \infty$ has been introduced only recently by Metzner and Vollhardt. ${ }^{13}$ In this limit it is vital to scale the kinetic energy correctly to obtain a nontrivial Hamiltonian. The application of this approach to the study of correlated fermion models has since made possible investigations which are not tractable in any finite dimension. ${ }^{14}$ In the spirit of mean-field theories for classical models, we may define the exact solution of a fermionic model in $Z=\infty$ to be a mean-field theory for this model. In view of the existence of a small parameter, $1 / Z$, which can be used for expansions around the mean-field limit, we call this a controlled mean-field theory, in contrast to mean-field theories where a systematic improvement cannot be made.

In this paper we will investigate the problem of disordered electrons in the limit $Z \rightarrow \infty$. The exact expression for the one-particle Green function, and thus the DOS, is derived. Thereby we obtain a controlled mean-field theory for these quantities in the sense described above. The self-consistent equation determining the Green function is found to be identical to that obtained by use of the CPA in the limit $Z \rightarrow \infty$. Hence, we find that for $Z=\infty$, the CPA yields exact results for the one-particle properties over the whole parameter range of impurity concentrations and scattering strengths. This explains why the CPA works so well even for intermediate values of the parameters in $d=3$. Apparently the dimension $d=3$ is already rather high ( $Z=6$ for a simple-cubic lattice), such that $1 / Z$ is a sufficiently small (expansion) parameter.

It should be noted that the importance of the small parameter $Z^{-1}$ for the CPA was already recognized in an important paper by Schwartz and Siggia. ${ }^{15}$ These authors asked precisely the question discussed above, namely whether it is possible to identify a parameter such that the CPA is the first term in a systematic expansion in this parameter. Investigating the moments of the electronic 
DOS, they found that, in addition to the impurity concentration and the potential strength, these moments depend on the "hidden" parameter $Z^{-1}$, and that $Z^{-1}$ governs the size of the corrections to the CPA moments. However, by studying only the moments, Schwartz and Siggia $^{15}$ did not investigate the full problem itself in the limit $Z \rightarrow \infty$; most importantly, they did not scale the kinetic energy (i.e., the bandwidth) properly, which is essential to obtain a nontrivial Hamiltonian for $Z=\infty$. Hence their approach is not a consistent expansion in $Z^{-1}$. This has direct consequences for the perturbation expansion (diagrammatic classification). They emphasized that $Z^{-1}$ was a relevant small parameter only for the moments of the self-energy and the DOS, while we can now show that the CPA, and the concept of the approximation itself, becomes exact for $Z \rightarrow \infty$. The latter can be proved in several different ways (expansion in the disorder or in the hopping) and is valid for arbitrary lattices (e.g., hypercubic, Bethe) and disorder distributions (e.g., box-shaped, binary alloy).

The paper is organized as follows. In Sec. II the disorder model and the different diagrammatic perturbation techniques for its investigation are introduced. In Sec. III the diagrammatic simplifications occurring in the limit $Z \rightarrow \infty$ are discussed. The connection with the CPA is clarified in Sec. IV. The results thus obtained are evaluated explicitly in Sec. V for the box-shaped and binaryalloy distributions on the Bethe lattice. A discussion (Sec. VI) ends the presentation.

\section{THE MODEL OF DISORDER}

The basis of our investigations is a tight-binding Hamiltonian with diagonal disorder, ${ }^{16}$

$$
H=\sum_{i, j} t_{i j} c_{i}^{\dagger} c_{j}+\sum_{i} \varepsilon_{i} c_{i}^{\dagger} c_{i}
$$

where $c_{i}^{\dagger}\left(c_{i}\right)$ are the creation (destruction) operators for fermions on site $i$. The hopping integral between the sites $i$ and $j$ is $t_{i j}$, which equals $t$ in case the sites $i$ and $j$ are nearest neighbors, and equals zero otherwise. The energy $\varepsilon_{i}$ is a stochastic variable drawn from some distribution function $P\left(\varepsilon_{1}, \varepsilon_{2}, \ldots, \varepsilon_{N}\right)$, where $N$ is the number of lattice sites. A physical quantity, $X$, which is a function of all site energies, is averaged with respect to this distribution as

$$
\begin{array}{r}
\bar{X} \equiv\left\langle\left\langle X\left(\varepsilon_{1}, \ldots, \varepsilon_{N}\right)\right\rangle\right\rangle=\int d \varepsilon_{1} \cdots \int d \varepsilon_{N} X\left(\varepsilon_{1}, \ldots, \varepsilon_{N}\right) \\
\times P\left(\varepsilon_{1}, \ldots, \varepsilon_{N}\right) .
\end{array}
$$

Concerning the distribution function, we assume that there are no correlations between the sites and that the distribution function is site independent. In this case the distribution factorizes as

$$
P\left(\varepsilon_{1}, \ldots, \varepsilon_{N}\right)=\prod_{i=1}^{N} P\left(\varepsilon_{i}\right) .
$$

The choice of $P(\varepsilon)$ will be postponed until explicit examples are treated. Particular cases with which we shall work are the "box" distribution,

$$
P(\varepsilon)=\frac{\Theta(W / 2-|\varepsilon|)}{W},
$$

where $\Theta(z)$ is the unit-step function, and the "binaryalloy" distribution,

$$
P(\varepsilon)=c \delta(\varepsilon-W / 2)+(1-c) \delta(\varepsilon+W / 2),
$$

where $\delta(z)$ is the $\delta$-function and $c$ determines the relative weight of the $\delta$-functions, i.e., $c$ is the concentration of impurities. In both cases, $W$ is the width of the distribution. From a calculational point of view, the latter distribution is particularly convenient.

For the calculation of physical quantities, the Greenfunction formalism is well suited. The equation of motion for the Green function $G_{i j}(\omega)$ is given by ${ }^{5}$

$$
\left(\omega-\varepsilon_{i}\right) G_{i j}(\omega)-\sum_{k} t_{i k} G_{k j}(\omega)=\delta_{i j},
$$

where $\omega$ is the frequency.

\section{A. Propagator versus locator formalism}

Within perturbation theory, the equation of motion (6) can be solved by two different approaches. ${ }^{4,5}$

(i) The random energy may be chosen as the expansion parameter. In this case we work in the so-called "propagator formalism." Solution of Eq. (6) with $\varepsilon_{i}=0$ provides a Green function, denoted $G_{i j}^{0}(\omega)$, which is called the bare propagator. The Green function $G_{i j}(\omega)$, called the full propagator here, is found using the iteration

$$
G_{i j}(\omega)=G_{i j}^{0}(\omega)+\sum_{k} G_{i k}^{0}(\omega) \varepsilon_{k} G_{k j}(\omega)
$$

(ii) The hopping integral can be chosen as the expansion parameter. Then we work in the locator formalism. Solution of Eq. (6) with $t_{i j}=0$ provides a Green function, denoted $g_{i}^{0}(\omega)$, which is called the bare locator. The Green function $G_{i j}(\omega)$ is found from

$$
G_{i j}(\omega)=g_{i}^{0}(\omega) \delta_{i j}+g_{i}^{0}(\omega) \sum_{k} t_{i k} G_{k j}(\omega)
$$

The diagonal element $G_{i i}(\omega)$ is called the full locator and is denoted $g_{i}(\omega)$.

The averaging procedure, Eq. (2), can also be performed in two different ways.

(a) Averaging can be carried through directly in Eqs. (7) and (8). In the propagator formalism the self-energy is defined as

$$
\sum_{k} \bar{\Sigma}_{i k}(\omega) \bar{G}_{k j}(\omega)=\left\langle\left\langle\varepsilon_{i} G_{i j}(\omega)\right\rangle\right\rangle
$$

where an overbar denotes an averaged quantity, so that we can write a Dyson equation for the averaged Green function $\bar{G}_{i j}(\omega)$ as

$$
\bar{G}_{i j}(\omega)=G_{i j}^{0}(\omega)+\sum_{k, l} G_{i k}^{0}(\omega) \bar{\Sigma}_{k l}(\omega) \bar{G}_{l j}(\omega)
$$

Since every diagram occurs only once in $\bar{G}_{i j}, \bar{\Sigma}_{i j}$ contains 
only irreducible diagrams with respect to $G_{i j}^{0}$. On the other hand, one can define - in the locator formalism in analogy to Eq. (10), a Dyson-like equation, ${ }^{17}$

$$
\bar{G}_{i j}(\omega)=\bar{\zeta}_{i j}(\omega)+\sum_{k, l} \bar{\zeta}_{i k}(\omega) t_{k l} \bar{G}_{l j}(\omega),
$$

in which $\bar{\zeta}_{i j}(\omega)$ is the so-called self-resolvent. It contains all irreducible diagrammatic contributions with respect to $t_{i j}$. All problems regarding the actual calculation of $\bar{G}_{i j}(\omega)$ are then shifted to the self-energy or the selfresolvent, respectively. All further investigations focus on these quantities. One problem is overcounting. This is a direct consequence of the summation over all sites in Eqs. (10) and (11). If one takes for the self-energy (or the self-resolvent) the set of all irreducible diagrams, then some classes of diagrams in $G_{i j}$ are overcounted. Therefore the diagrammatic definitions of the self-energy and the self-resolvent must be changed in such a way that this overcounting does not take place. This correction procedure $^{17}$ is known as multiple-occupancy correction (MOC).

(iii) One can try to order the diagrams in such a way that, when the averaging is carried through, no overcounting takes place. This implies that restrictions must be imposed on the summations over the sites in Eqs. (7) and (8) before averaging takes place. This technique is known as the renormalized perturbation expansion (RPE). ${ }^{18,5}$ Since the RPE is particularly useful for the Bethe lattice, we will study this technique in more detail in the next subsection.

\section{B. The RPE locator formalism}

As discussed above, we must regroup the diagrams if we want to apply the RPE. To this end, we write an equation for the full locator with base site $i, g_{i}(\omega)$, in analogy to the Dyson equation in the propagator formalism,

$$
g_{i}(\omega)=g_{i}^{0}(\omega)+g_{i}^{0}(\omega) \sigma_{i}(\omega) g_{i}(\omega),
$$

where $\sigma_{i}(\omega)$ is the site-dependent self-energy. When the
Green function is expressed in terms of the full locators, the first signs of the RPE become visible:

$$
\begin{aligned}
G_{i j}(\omega)= & g_{i}(\omega) \delta_{i j}+g_{i}^{-(j)}(\omega) t_{i j} g_{j}(\omega) \\
& +\sum_{k} g_{i}^{-(j, k)}(\omega) t_{i k} g_{k}^{-(j)}(\omega) t_{k j} g_{j}(\omega)+\cdots .
\end{aligned}
$$

Here a superscript representing one or more sites with an overall minus sign implies that the locator does not depend on the energy of these particular sites. Thus, for example, the expansion of $g_{j}^{-(i)}$ in terms of bare locators contains all possible bare locators except $g_{i}^{0}$. This notation will later prove to be useful, since diagrammatic simplifications-for example, in the case of the Bethe lattice-can be recognized easily. In the interpretation of this expression some care has to be taken. In spite of the exclusion of site $k$ from the locator based at site $i$, $g_{i}^{-(j, k)}$, correlations with respect to the averaging procedure given in Eq. (2) between the two locators $g_{i}^{-(j, k)}$ and $g_{k}^{-(j)}$ do exist. This subtlety will also be of importance in a diagrammatic representation of the equations.

It is also desirable to have an expression for the selfenergy $\sigma_{i}(\omega)$. Making use of $G_{i i}(\omega)=g_{i}(\omega)$ and Eqs. (12) and $(8)$, we find

$$
\sigma_{i}(\omega) g_{i}(\omega)=\sum_{k} t_{i k} G_{k i}(\omega)
$$

In terms of full locators, this reads

$$
\begin{aligned}
\sigma_{i}(\omega)= & \sum_{k} t_{i k} g_{k}^{-(i)}(\omega) t_{k i} \\
& +\sum_{k, l} t_{i k} g_{k}^{-(i, l)}(\omega) t_{k l} g_{l}^{-(i)}(\omega) t_{l i}+\cdots .
\end{aligned}
$$

It thus becomes clear that the general quantities of the theory are the full locator and the self-energy with the above-mentioned exclusion of any number of sites. These are indicated by $g_{i}^{-\left(j_{1}, \ldots, j_{n}\right)}$ and $\sigma_{i}^{-\left(j_{1}, \ldots, j_{n}\right)}$, respective1y. The equations connecting them are generalizations of Eqs. (12) and (15), i.e.,

$$
\begin{aligned}
& g_{i}^{-\left(j_{1}, \ldots, j_{n}\right)}(\omega)=g_{i}^{0}(\omega)+g_{i}^{0}(\omega) \sigma_{i}^{-\left(j_{1}, \ldots, j_{n}\right)}(\omega) g_{i}^{-\left(j_{1}, \ldots, j_{n}\right)}(\omega), \\
& \sigma_{i}^{-\left(j_{1}, \ldots, j_{n}\right)}(\omega)=\sum_{k} t_{i k} g_{k}^{-\left(i, j_{1}, \ldots, j_{n}\right)}(\omega) t_{k i}+\sum_{k, l} t_{i k} g_{k}^{-\left(i, l, j_{1}, \ldots, j_{n}\right)}(\omega) t_{k l} g_{l}^{-\left(i, j_{1}, \ldots, j_{n}\right)}(\omega) t_{l i}+\cdots .
\end{aligned}
$$

Formally, these equations equal Eqs. (12) and (15) for $n=0$. Note that the dependence of the problem on the underlying lattice enters only through Eq. (17) for the self-energy.

In order to gain a better insight into the simplifications arising in the limit $Z \rightarrow \infty$ and on the Bethe lattice, we express the equations obtained so far in diagrammatic language. The diagrammatic elements are depicted in Fig. 1. The bare (full) locator $g_{i}^{0}\left(g_{i}\right)$ consists of a vertical, dotted (solid) line terminating at site $i$ indicated by a cross. The hopping matrix elements $t_{i j}$ are represented by directed (see arrow), horizontal solid lines leading from site $i$ to site $j$ (these sites are not explicitly indicated). The self-energy $\sigma_{i}$ consists of a directed line with a dot marked $i$. In Figs. 2(a), and 2(b), we see the diagrammatic representation of Eqs. (12) and (15), respectively. In the figure the summation over internal site variables is assumed. The subtlety regarding the correlations between different full locators is illustrated in Fig. 2(c). This diagram is a typical example of an element contained in the second expansion term on the right-hand side of Eq. (15). This makes it clear that there is a correlation between the two locators, namely through some site $p$. 

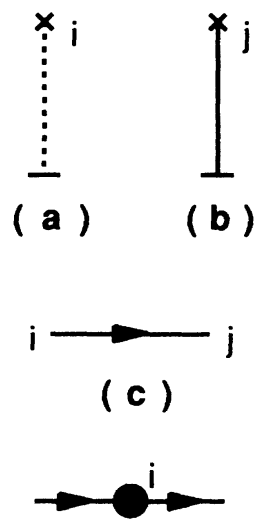

\section{(d)}

FIG. 1. Diagrammatic elements within the locator formalism: (a) bare locator, (b) full locator, (c) hopping integral, and (d) self-energy.

Having discussed the general diagrammatic representation, it remains to explain how the averaging is done. At this level it can only be performed in a formal way. We indicate the general, averaged element with $n$ excluded sites by the superscript [ $n]$; explicitly, we have

$$
\begin{aligned}
& \bar{g}^{[n]}=\left\langle\left\langle g_{i}^{-\left(j_{1}, \ldots, j_{n}\right)}\right\rangle\right\rangle, \\
& \bar{\sigma}^{[n]}=\left\langle\left\langle\sigma_{i}^{-\left(j_{1}, \ldots, j_{n}\right)}\right\rangle\right\rangle .
\end{aligned}
$$

Thus, the price paid for avoiding the MOC procedure is that one is left with a whole set of averaged self-energies, instead of just one.

In summary, we see that in the RPE locator formalism the equation of motion is written as Eqs. (16) and (17). If these are solvable, i.e., if the general elements $g_{i}{ }^{-\left(j_{1}, \ldots, j_{n}\right)}$ and $\sigma_{i}^{-\left(j_{1}, \ldots, j_{n}\right)}$ can be calculated, averaging can be performed by Eqs. (18) and (19). The averaged full Green function, $\bar{G}_{i j}(\omega)$, is found directly by averaging of Eq. (13).

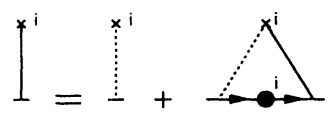

(a)

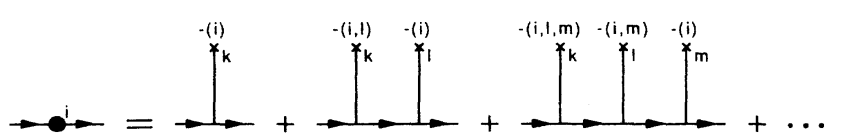

(b)

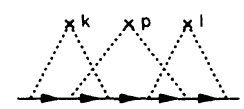

(c)

FIG. 2. (a) Dyson equation for the full locator, (b) expansion of the self-energy in full locators, and (c) diagrammatic element with correlated locators contained in the second diagram in the expansion of the self-energy, shown in (b).

\section{DIAGRAMMATIC SIMPLIFICATIONS}

The diagrammatic structure of the theory depends crucially on the geometry of the underlying lattice. Comparison of the hypercubic lattice with the Bethe lattice ${ }^{18}$ shows that the latter leads to much simpler mathematical expressions. In both cases the limit $Z \rightarrow \infty$ can be performed. Metzner and Vollhardt ${ }^{13}$ have shown that in this limit the hopping integral between nearest neighbors, $t$, has to be rescaled according to

$$
t=\frac{\tilde{t}}{\sqrt{Z}}, \quad \tilde{t}=\text { const }
$$

for the kinetic energy to remain finite for $Z \rightarrow \infty$. Hence, $t$ is of order of $1 / \sqrt{Z}$. All diagrams can thus be systematically classified according to the power of $1 / \sqrt{Z}$ that they contribute.

Let us first find out which diagrams remain in the limit $Z \rightarrow \infty$. The expansion of the full locator [Eq. (12)] is given in Fig. 3(a). In the limit $Z \rightarrow \infty$ the different selfenergies become uncorrelated. To illustrate this point, we have to take a look at two diagrams contained in the third expansion term of $g_{i}$. The first, given in Fig. 3(b), contains four hopping integrals and one free site $k$; the latter is a direct neighbor of the fixed site $i$. The total contribution is $(1 / \sqrt{Z})^{4} Z=Z^{-1}$, resulting from four hopping events, multiplied by $Z$, the number of possibilities for $k$ to be a neighbor of $i$. This diagram can thus be neglected in the limit $Z \rightarrow \infty$. The second, given in Fig. 3 (c), contains four hopping integrals too, but has two free sites $k$ and $l$ that are direct neighbors of $i$. Therefore there is an extra factor $Z$ compared to the first case, which implies that the contribution is of order $Z^{0}=1$. This diagram remains in the limit $Z \rightarrow \infty$. This example illustrates that all diagrams having correlations between the different self-energies are at least of order $Z^{-1}$ and do not survive in the limit $Z \rightarrow \infty$.

The arguments that led to the conclusion that different $\sigma_{i}$ are uncorrelated in the expansion of a single full locator $g_{i}$ are also valid for the self-energies $\sigma_{i}$ and $\sigma_{j}$ in the expansion of a product of full locators in which $g_{i}$ and $g_{j}$ are present. These correlations may thus also be neglected in the limit $Z \rightarrow \infty$. This is important for instance in the expansion of $\sigma_{i}$ itself [Eq. (15), or Fig. 2(b)].

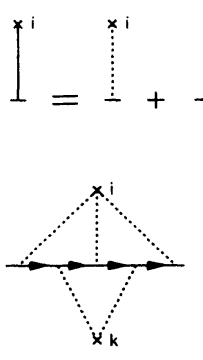

(b)

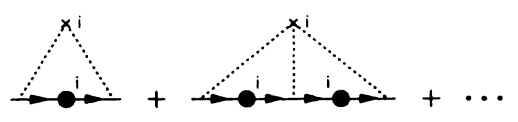

(a)

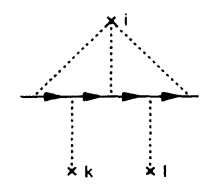

(c)
FIG. 3. (a) Expansion of the full locator in terms of selfenergies and bare locators, (b) diagrammatic contribution of order $1 / Z$, and (c) diagrammatic contribution of order 1 . 


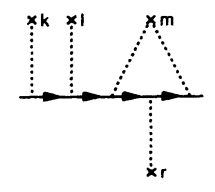

(a)

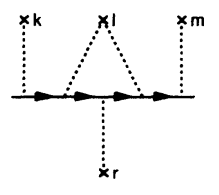

(b)

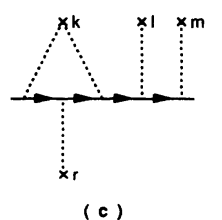

(c)
FIG. 4. Third term in the expansion of the self-energy in Fig. 2 expanded into bare locators. As a result of RPE, the site $r$ in diagrams $(a)-(c)$ is subject to different constraints (see text).

There are more simplifications in the limit $Z \rightarrow \infty$. These are connected with the negative site superscripts. To illustrate this point, we can take a look at Fig. 4. The third expansion term of $\sigma_{i}$ [Eq. (15), or Fig. 2(b)] is expanded into bare locators. The negative site superscripts on the full locators have implications for the site marked $r$ in Figs. 4(a) $-4(\mathrm{c})$. In general, we have the following situation. The site $r$ may be equal to site $k$ or $l$ in Fig. 4(a). The site $r$ may be equal to site $k$, but not equal to site $m$ in Fig. 4(b); and in Fig. 4(c), site $r$ may not be equal to site $l$, and not equal to site $m$ either. In the limit $Z \rightarrow \infty$, however, a diagram like Fig. 4(b), with site $r$ equal to $m$, or Fig. 4(c), with site $r$ equal to site $l$ or $m$ (both diagrams are forbidden by the rules of RPE) would not contribute because they are at least of order $Z^{-1}$. The restrictions with respect to the negative site superscripts, which are a direct consequence of the RPE, may therefore be relaxed. In other words, by including certain (formally forbidden) diagrams, which do not contribute for $Z \rightarrow \infty$, inconvenient restrictions may be dropped, thus yielding a simpler theory. This relaxation does not apply to the base site of a full locator. It is thus not allowed to make site $l$ equal to $m$ in Fig. 4(c); the diagram that arises in this case already contributes and would then be counted twice. Hence, in the limit $Z \rightarrow \infty$, $g_{k}^{-(i, l, m)}$ must be interpreted as $g_{k}$ with $k \notin\{i, l, m\}$. We shall simply call this phenomenon "index relaxation." Of course, the simplifications just discussed also hold for the Green function given in Eq. (13).

\section{A. Averaging and the limit $Z \rightarrow \infty$}

What are the consequences of the simplifications discussed thus far? Owing to index relaxation, all the averaged full locators, $\bar{g}^{[n]}$, are now equal, i.e., independent of $n$; likewise, all $\bar{\sigma}^{[n]}$ are the same for arbitrary $n$. They can simply be indicated by $\bar{g}$ and $\bar{\sigma}$, respectively. The full locator can now easily be averaged in the limit $Z \rightarrow \infty$ using Eq. (12) instead of the more complicated Eq. (16). Furthermore, the different $\sigma_{i}$ can be treated as uncorrelated as stated above; hence,

$$
\left\langle\left\langle g_{i}(\omega)\right\rangle\right\rangle=\left\langle\left\langle g_{i}^{0}(\omega)+g_{i}^{0}(\omega)\left\langle\left\langle\sigma_{i}(\omega)\right\rangle\right\rangle g_{i}(\omega)\right\rangle\right\rangle \text {. }
$$

Expanding Eq. (21) and making use of the decorrelation between the different $\sigma_{i}$ gives rise to a geometric series in $g_{i}^{0}(\omega) \bar{\sigma}(\omega)$. Summation and substitution of $g_{i}^{0}(\omega)=\left(\omega-\varepsilon_{i}\right)^{-1}$ leads to

$$
\bar{g}(\omega)=\left\langle\left\langle\frac{1}{\omega-\varepsilon_{i}-\bar{\sigma}(\omega)}\right\rangle .\right.
$$

Owing to index relaxation, the averaged self-energy, $\bar{\sigma}(\omega)$, is directly obtained from Eq. (15) rather than from the more complicated Eq. (17). Making further use of the decorrelation of internal self-energies inside $g_{i}^{-\left(j_{1}, \ldots, j_{n}\right)}$, we obtain

$$
\bar{\sigma}(\omega)=\sum_{k(\neq i)} t_{i k}\left\langle\left\langle g_{k}(\omega)\right\rangle t_{k i}+\sum_{l(\neq i)} \sum_{k(\neq i, l)} t_{i k}\left\langle\left\langle g_{k}(\omega)\right\rangle\right\rangle t_{k l}\left\langle\left\langle g_{l}(\omega)\right\rangle\right\rangle t_{l i}+\cdots\right.
$$

This equation can be condensed further by introducing the number of closed, non-self-intersecting paths of $m$ steps, $\xi(m)$, via

$$
\bar{\sigma}(\omega)=\sum_{m=1}^{\infty} \xi(m+1)[\bar{g}(\omega)]^{m} t^{m+1} .
$$

Thereby it becomes apparent how the lattice structure enters into the calculation of the self-energy. The same can be done for the full Green function, $\bar{G}_{i j}(\omega)$. Using the function $\xi_{i j}(m)$, giving the number of non-selfintersecting paths from site $i$ to site $j$ in exactly $m$ steps, one obtains

$$
\bar{G}_{i j}(\omega)=\sum_{m=1}^{\infty} \xi_{i j}(m)[\bar{g}(\omega)]^{m+1} t^{m}
$$

To recapitulate, we see that for $Z \rightarrow \infty$ the general problem, expressed by Eqs. (13), (16), and (17), together with the averaging in Eqs. (18) and (19), simplifies to Eqs. (25), (22), and (24). As such, the latter equations are the central ones of this paper.

\section{B. Corrections in $1 / Z$}

The next step is to construct an expansion in the parameter $Z^{-1}$. The first correction to the limit $Z \rightarrow \infty$ is to take all diagrams into account up to the order $Z^{-1}$. In Fig. 5(a) the general diagrammatic element is depicted that must be part of any diagram to ensure that its contribution is reduced by at least a factor $1 / Z$ compared to the same diagram having, instead, a bare hopping at this place. One example was already encountered in Fig. 3(b). Two more are shown in Figs. 5(b) and 5(c).

Furthermore, the implementation of the index relaxation is changed. For example, in Fig. 4 we see that, if we make site $r$ in Fig. 4(b) equal to site $m$, a diagram of order $1 / Z$ results. This formally inadmissible diagram will then give a contribution to the order of interest, $1 / Z$. Any further violation of the RPE restrictions will not change the result in the order $1 / Z$. Thus we may conclude that the index relaxation only applies to diagrams that are already of order $1 / Z$.

Of great importance for the calculation of the $1 / Z$ 


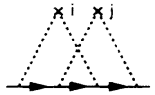

(a)

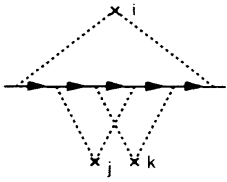

(b)

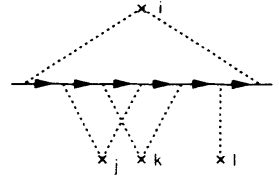

(c)
FIG. 5. Diagrammatic corrections in $1 / Z$ : (a) general element contained in any diagram contributing at least to order $1 / Z$; (b) example of a diagram of order $1 / Z$, which only contributes when the sites $i, j$, and $k$ form a triangle on the lattice; (c) same as (b), but the sites $i, j, k$, and $l$ have to form a square on the lattice.

corrections is the underlying lattice structure. Not every diagram of order $1 / Z$ is compatible with the lattice. For example, the diagram in Fig. 3(b) contributes for every lattice structure. The diagram in Fig. 5(c) contributes on the hypercubic lattice, but not on a Bethe lattice, because the latter only allows for self-retracing closed paths. The diagram shown in Fig. 5(b) contributes to neither casesuch a diagram only contributes when the site $i, j$, and $k$ form a triangle on the lattice. It is therefore not possible to write an equation, such as Eq. (22), in a latticeindependent form from which the averaged quantities, correct in the order $1 / Z$, can be calculated.

\section{The Bethe lattice}

In the following we will assume the underlying lattice to be a Bethe lattice. Let us define the connectivity by $K=Z-1$. The absence of closed loops in this case leads to great simplifications in the equations of motion; ${ }^{5}$ this was already exploited by Abou-Chakra et al. ${ }^{18}$ Therefore, in every diagonal quantity, e.g., the locator and the selfenergy, the electron must follow a self-retracing path. Note, however, that the sublety concerning the correlations between different full locators, discussed in Sec. II B in the context of Fig. 2(c), remains. The expression for the Green function then simplifies to

$$
\begin{aligned}
G_{i j}(\omega)= & g_{i}(\omega) \delta_{i j}+g_{i}^{-(j)}(\omega) t_{i j} g_{j}(\omega) \\
& +\sum_{k} g_{i}^{-(k)}(\omega) t_{i k} g_{k}^{-(j)}(\omega) t_{k j} g_{j}(\omega)+\cdots,
\end{aligned}
$$

where for every pair of fixed sites $i$ and $j$ only one term of the expansion contributes. The expression for the full locator [Eq. (12)] does not change, but the self-energy reduces to

$$
\sigma_{i}(\omega)=\sum_{k} t_{i k} g_{k}^{-(i)}(\omega) t_{k i},
$$

i.e., only the first term in Fig. 2(b) remains.

Even for the Bethe lattice, with general connectivity $K$, the DOS is not exactly calculable for the disordered electron problem. However, the problem can be simplified further by taking the limit of infinite branching: $K \rightarrow \infty$. The theory discussed so far is directly applicable in this situation. The rescaled hopping is denoted in the form $\widetilde{t}^{2}=K t^{2}$, with $\widetilde{t}$ finite. In this limit Eqs. (22) and (27) must be solved taking into account the index relaxation. This yields

$$
\begin{aligned}
& \bar{g}_{\infty}(\omega)=\left\langle\left\langle\frac{1}{\omega-\varepsilon_{i}-\bar{\sigma}_{\infty}(\omega)}\right\rangle,\right. \\
& \bar{\sigma}_{\infty}(\omega)=\tilde{t}^{2} \bar{g}_{\infty}(\omega) .
\end{aligned}
$$

The subscript $\infty$ is meant to distinguish between the results for infinite branching and those including $K^{-1}$ corrections. This set can also be derived directly from Eqs. (22) and (24). In this case one must realize that for the Bethe lattice the number of non-self-intersecting closed loops is given by $\xi(m)=(K+1) \delta_{m, 2}$. Substitution into Eq. (24) then brings us directly to Eq. (28). The averaged, full Green function can also directly be found using $\xi(m)=\delta_{m,|i-j|} ;$ this yields

$$
\bar{G}_{i j}(\omega)=\bar{g}(\omega)[t \bar{g}(\omega)]^{|i-j|} .
$$

For the Bethe lattice it is possible to obtain explicit equations for the $K^{-1}$ corrections. However, this calculation is rather lengthy and is given in Appendix A; here we merely state the result. Up to order $K^{-2}$ in perturbation theory, one obtains

$$
\begin{aligned}
& \bar{g}=\bar{g}^{[1]}+\frac{\widetilde{t}^{2}}{K} \bar{g}_{\infty}\left\langle\left\langle\left[\omega-\varepsilon_{i}-\bar{\sigma}_{\infty}(\omega)\right]^{-2}\right\rangle\right\rangle+O\left(K^{-2)},\right. \\
& \bar{g}^{[1]}=\bar{g}_{\infty}+\frac{\widetilde{t}^{4}}{K} \frac{\left.\left\{\left\langle\left[\omega-\varepsilon_{i}-\bar{\sigma}_{\infty}(\omega)\right]^{-2}\right\rangle\right\rangle-\bar{g}_{\infty}^{-2}\right\}\left\langle\left\langle\left[\omega-\varepsilon_{i}-\bar{\sigma}_{\infty}(\omega)\right]^{-3}\right\rangle\right\rangle}{1-\widetilde{t}^{2}\left\langle\left\langle\left[\omega-\varepsilon_{i}-\bar{\sigma}_{\infty}(\omega)\right]^{-2}\right\rangle\right\rangle}+O\left(K^{-2}\right) .
\end{aligned}
$$

\section{THE CONNECTION TO THE CPA}

The coherent potential approximation ${ }^{6-10}$ (CPA) is usually formulated in the propagator formalism. The CPA is a single-site approximation ${ }^{17,10}$ that embeds the individual scatterers in an effective medium. This effective medium is determined self-consistently by the requirement that the scattering from a given site in the medium equals the scattering of the medium as a whole. As a consequence, the self-energy $\bar{\Sigma}(\omega)$ is a diagonal ma- 


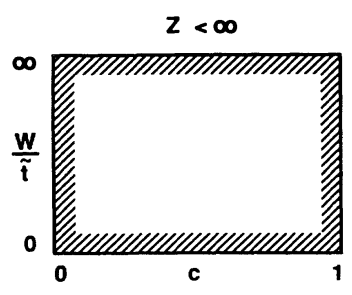

(a)

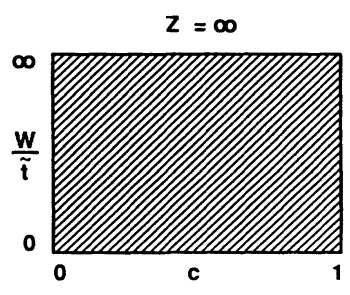

(b)
FIG. 6. The CPA is exact in the hatched region of parameters (disorder vs concentration). (a) $Z<\infty$; the CPA becomes exact for low $(c \rightarrow 0)$ and high $(c \rightarrow 1)$ concentrations of impurities, as well as for weak scattering $(W \rightarrow 0)$ and in the atomic limit $(\tilde{t} \rightarrow 0)$. (b) $Z=\infty$; the CPA is exact for every value of $c$ and $W$.

trix

$$
\bar{\Sigma}_{i i}(\omega)=\left\langle\left\langle\frac{\varepsilon_{i}}{1-\left[\varepsilon_{i}-\bar{\Sigma}_{i i}(\omega)\right] \bar{G}_{i i}(\omega)}\right\rangle .\right.
$$

The CPA is known to be exact in several limiting re- gimes, i.e., for low $(c \rightarrow 0)$ and high $(c \rightarrow 1)$ concentrations of impurities, as well as for weak scattering $(W \rightarrow 0)$ and in the atomic limit $(t \rightarrow 0)$. As pointed out by Leath, ${ }^{19}$ the region of parameters where the CPA is obtained by perturbation theory can be summarized graphically as shown in Fig. 6(a). [Note, that for $t=$ const and $W \rightarrow \infty$, an exact solution does not exist; hence the hatched region at the top of Fig. 6(a) only refers to an expansion around the atomic limit, not to a general perturbation expansion in $1 / W$.] In the interior region the CPA gives a simple and analytic interpolation, which often yields surprisingly good quantitative results, even in $d=3$ and lower dimensions, particularly if cluster effects can be neglected.

Let us formulate the CPA using the RPE locator formalism. This is, in fact, much easier to do in this than in any other formalism, because the RPE effectively isolates contributions from single sites. From Eq. (15) it is seen that $\sigma_{i}$ does not contain the local potential $\varepsilon_{i}$. Therefore, by expansion of Eq. (12) it is clear that $\varepsilon_{i}$ appears only in $g_{i}$. The single-site approximation implies that the averaging is decoupled at every place where $\varepsilon_{i}$ appears in the expansion of $g_{i}$. Hence,

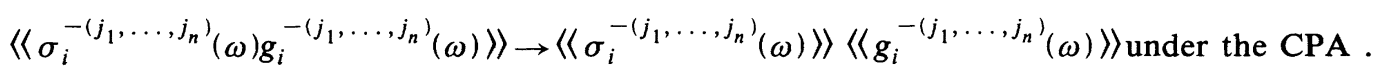

Thus, the CPA is given by a whole hierarchy of equations rather than a single one such as Eq. (31). This is why, to our knowledge, this formulation has never been used before to derive the CPA. We apply this decorrelation approximation to Eqs. (16) and (17). In Eq. (16) it is possible, as we did in Eq. (21), to expand $g_{i}^{-\left(j_{1}, \ldots, j_{n}\right)}$ into a geometric series. Resummation yields

$$
\bar{g}^{[n]}=\left\langle\left\langle\frac{1}{\omega-\varepsilon_{i}-\bar{\sigma}^{[n]}}\right\rangle\right\rangle=\left\langle\left\langle g_{i}^{0}\left(\omega-\bar{\sigma}^{[n]}\right)\right\rangle\right\rangle, \text { for all } n .
$$

The latter expression is similar in structure to that obtained by formulating the CPA in the propagator formalism. ${ }^{6,7,10}$ It connects the averaged full Green function to the averaged bare Green function at a displaced energy. The CPA hierarchy is closed by the coupling between $\bar{\sigma}^{[n]}$ and $\bar{g}^{[m]}, m>n$, determined by the lattice structure. Using Eq. (32), it follows from Eq. (17) that

$$
\left\langle\left\langle\sigma_{i}^{-\left(j_{1}, \ldots, j_{n}\right)}(\omega)\right\rangle\right\rangle=\sum_{k} t_{i k}\left\langle\left\langle g_{k}^{-\left(i, j_{1}, \ldots, j_{n}\right)}(\omega)\right\rangle\right\rangle t_{k i}+\sum_{k, l} t_{i k}\left\langle\langle g _ { k } ^ { - ( i , l , j _ { 1 } , \ldots , j _ { n } ) } ( \omega ) \rangle t _ { k l } \left\langle\left\langle g_{l}^{-\left(i, j_{1}, \ldots, j_{n}\right)}(\omega)\right\rangle t_{l i}+\cdots,\right.\right.
$$

which can be simplified to

$$
\bar{\sigma}^{[n]}(\omega)=\sum_{k\left(\neq j_{1}, \ldots, j_{n}\right)} t \bar{g}^{[n+1]}(\omega) t+\sum_{l\left(\neq i, j_{1}, \ldots, j_{n}\right)} \sum_{k\left(\neq i, k, j_{1}, \cdots, j_{n}\right)} t \bar{g}^{[n+2]}(\omega) t \bar{g}^{[n+1]}(\omega) t+\cdots .
$$

The exclusion of the sites $j_{1}, \ldots, j_{n}$ is due to closed paths that pass through $j_{1}, \ldots, j_{n}$ and appear in the calculation of $\bar{\sigma}^{[k]}$ with $k<n$. If we want to cast this into a form where specific sites do not appear, introduction of a loop operator $\xi^{[n]}(m)$ is necessary. Here, $\xi^{[0]}\left(m_{0}\right)$ simply gives the number of non-self-intersecting closed paths of $m_{0}$ steps on the given lattice; hence it equals the function $\xi\left(m_{0}\right)$ introduced in Eq. (24). Similarly, $\xi^{[1]}\left(m_{1}\right)$ gives the number of closed, non-self-intersecting paths of $m_{1}$ steps, with the additional restriction that the path does not intersect the path in $\xi^{[0]}\left(m_{0}\right)$. In general, $\xi^{[n]}\left(m_{n}\right)$ gives the number of closed, non-self-intersecting paths of $m_{n}$ steps, with the additional restriction that the paths does not intersect any of the paths in $\xi^{[k]}\left(m_{k}\right)$, with $k<n$, appearing in products of the form

$\xi^{[n]}\left(m_{n}\right) \xi^{[n-1]}\left(m_{n-1}\right) \times \cdots \times \xi^{[1]}\left(m_{1}\right) \xi^{[0]}\left(m_{0}\right)$.

This simplifies Eq. (35) to

$\bar{\sigma}^{[n]}(\omega)=\sum_{m=1}^{\infty} \xi^{[n]}(m+1)\left[\prod_{l=1}^{m} \bar{g}^{[n+l]}(\omega)\right] t^{m+1}$ 
In the limit $Z \rightarrow \infty$, Eq. (33) reduces to Eq. (22) as a result of the index relaxation discussed above. This equation, however, is exact in the limit $Z \rightarrow \infty$. The same applies to Eqs. (37) and (24). This is because the arguments which led to the index relaxation of $\bar{g}^{[n]}$ and $\bar{\sigma}^{[n]}$ are also directly applicable to $\xi^{[n]}(m)$, i.e., $\xi^{[n]}(m) \rightarrow \xi(m)$ as $Z \rightarrow \infty$. The results may, of course, be also directly understood on the basis of Eq. (32) itself, because it is an identity in the limit $Z \rightarrow \infty$.

Therefore, we have proven that the CPA becomes exact in the limit $Z \rightarrow \infty$. This result does not depend on the lattice structure. The analogous proof within the propagator formalism is given in Appendix B. We also see that the CPA and the limit $Z \rightarrow \infty$ are not equivalent. In fact, the results of the latter limit can be recovered from the CPA only by imposing additional simplifications (namely, index relaxation). Hence, approximating a system having a finite number of next neighbors by one with $Z=\infty$ is more restrictive than applying the CPA (also see Sec. V C).

This result clarifies why, for $Z<\infty$, e.g., finite dimensions on a regular lattice, the CPA can be so successful even for intermediate values of the parameters (concentration, scattering strength) where this can no longer be justified by perturbation theory. Indeed, our result shows that, for $Z=\infty$, the CPA is correct in the entire region of parameters, i.e., the hatched region in Fig. 6(a) expands for increasing $Z$ and eventually fills out the whole region, as shown in Fig. 6(b). Hence, the interpolation provided by the CPA, whose validity is a priori uncertain, is, in fact, seen to be controlled by the parameter $Z^{-1}$, because for $Z^{-1} \rightarrow 0$ this interpolation yields the exact result. It is therefore not surprising that, for $d=3$, where $Z^{-1} \simeq 0.17$ for a simple-cubic lattice (and even smaller for other lattices, e.g., a face-centered-cubic lattice), the CPA may give qualitatively and even quantitatively correct results.

The foundations of the CPA were investigated by Schwartz and Siggia, ${ }^{15}$ who recognized that the inverse of the number of nearest neighbors, $Z^{-1}$, is the relevant small parameter. They concluded that the CPA, in addition to being exact to first order in the concentration $c$ and to third order in the impurity potential $W$, retains just those contributions of higher order in $c$ and $W$ that are independent of $Z^{-1}$. However, they also stated that $Z^{-1}$ is a relevant parameter only for the moments of the self-energy and DOS. Therefore they concluded that it was not possible to make any specific statements about the validity of the CPA. In fact, these authors did not base their conclusions on the explicit calculation of the diagrams contributing to the CPA, but on an interpretation of the limit $Z \rightarrow \infty$ as being equivalent to the selfconsistent single-site approximation. Since they did not scale the kinetic energy with $1 / \sqrt{Z}$ (in their case, it decreases proportionally to $\sqrt{Z}$, such that the hopping becomes irrelevant), a diagrammatic summation will not lead to the CPA. The fact that the CPA becomes exact in the limit $Z \rightarrow \infty$, using the correct scaling of the hopping amplitude, was already anticipated by MüllerHartmann ${ }^{20}$ and by Czycholl. ${ }^{21}$

Previous extensions of the CPA (such as, for example, in Refs. 22 and 23, and references therein) were obtained by treating an $n$-site cluster exactly and embedding it into the effective medium in some self-consistent way. There is no small parameter in such a procedure and, hence, the extension is not systematic on a perturbational level. This in contrast to the extension of the limit $K \rightarrow \infty$ in the small parameter $1 / K$, Eq. (30). Since it is a perturbational extension, it is systematic and controlled. Of course, it is only valid as long as perturbation theory holds, e.g., away from the band edges, where, according to Eq. (30), the perturbation theory diverges. This can only be cured if the $1 / K$ corrections are included on a self-consistent level.

\section{APPLICATIONS: CHOICE OF THE DISORDER DISTRIBUTION}

The DOS, $N(\omega)$, is found from the full locator by

$$
N(\omega)=-\frac{1}{\pi} \operatorname{Im}[\bar{g}(\omega)] \text {. }
$$

We shall calculate $N(\omega)$ for the Bethe lattice in the limit $K \rightarrow \infty$, as well as the corrections to this result of order $1 / K$. We will do so both for the box distribution [Eq. (4)] and for the binary-alloy model [Eq. (5)] . The DOS for free electrons on a Bethe lattice is

$$
N(\omega)=\frac{1}{2 \pi} \frac{K(K+1)\left(4-\omega^{2}\right)^{1 / 2}}{(K+1)^{2}-K \omega^{2}}
$$

for general $K$. In this equation, and in the rest of this section, all energies are scaled with $\tilde{t}$. In this case the bandwidth is equal to 4 for all $K$, i.e., even in the limit $K \rightarrow \infty$. This is in contrast to the situation for the simple hypercubic lattice where, in infinite dimensions, ${ }^{13}$ the DOS is a Gaussian. It was already recognized by White and Economou $^{24}$ that one has to scale the hopping integral with $\sqrt{K}$ to obtain a sensible DOS in the limit $K \rightarrow \infty$. However, they made no further use of this observation.

The DOS for the Bethe lattice, expanded in $1 / K$, becomes

$N(\omega)=\frac{\left(4-\omega^{2}\right)^{1 / 2}}{2 \pi}\left[1+\frac{\omega^{2}-1}{K}+O\left[\frac{1}{K^{2}}\right]\right]$.

For infinite branching we have the Hubbard halfellipse. ${ }^{25,5}$ Note that this model DOS is often used as an additional approximation in combination with the CPA (see, for example, Ref. 8). For $K \geq 6$ the expansion in Eq. (40), including the $1 / K$ correction, is excellent, while for $2 \leq K \leq 5$ it is still good, at least in a qualitative sense. Only in the extreme case of $K=1$ is the result even qualitatively incorrect.

\section{A. The box distribution}

The DOS for the disordered system can be calculated analytically for the box distribution in the limit of infinite branching on the Bethe lattice. Performing the averaging integral, Eq. (2), with this distribution, Eq. (4), a closed equation for the full locator in the limit $K \rightarrow \infty, \bar{g}_{\infty}$ in Eq. (28), is obtained. Dividing the full locator into a real part, $\mathcal{R}$, and an imaginary part, $\mathcal{J}$, and making use of the fact that $\omega$ is real, we find 


$$
\begin{aligned}
& \exp (\mathcal{R})=\cos (\mathcal{J})+\frac{W^{2}}{2 \mathcal{J}} \sin (\mathcal{J}) \pm\left[\left[\cos (\mathcal{J})+\frac{W^{2}}{2 \mathcal{J}} \sin (\mathcal{I})\right]^{2}-1\right]^{1 / 2} \\
& \omega=\frac{\mathscr{R}}{W}+\frac{W}{2} \frac{\exp (2 \mathcal{R})-1}{\exp (2 \mathcal{R})-2 \exp (\mathcal{R}) \cos (\mathcal{J})+1} \\
& \bar{g}_{\infty}=(\mathcal{R}+i \mathcal{J}) / W
\end{aligned}
$$

The result for $N(\omega)$ is plotted in Fig. 7. The free case $(W=0)$ is compared to the disordered case for different values of the coupling parameter $W$. A band broadening is clearly observed; this is in agreement with early results by Brouers. ${ }^{26}$ Furthermore, the curve shows great resemblance with the half-ellipse for the free case, and the same polynomial decay is seen at the band edges. The band broadening can be explicitly calculated by solving Eq. (41) for $\mathcal{J}=0$. The bandwidth $B$ equals

$$
B=W+\frac{2}{W} \ln \left[1+\frac{W^{2}+W\left(W^{2}+4\right)^{1 / 2}}{2}\right]+\frac{4}{W+\left(W^{2}+4\right)^{1 / 2}},
$$

which is a monotonic function in $W$.

The $1 / K$ corrections are calculated using Eq. (30). A straightforward but lengthy calculation gives

$$
\bar{g}=\bar{g}_{\infty}+\left(\frac{4 \bar{g}_{\infty}}{\left[4\left(\bar{g}_{\infty}-\omega\right)^{2}-W^{2}\right]}-\frac{16\left(\bar{g}_{\infty}-\omega\right)\left\{4-\bar{g}_{\infty}^{2}\left[4\left(\bar{g}_{\infty}-\omega\right)^{2}-W^{2}\right]\right\}}{\left[4\left(\bar{g}_{\infty}-\omega\right)^{2}-W^{2}\right]^{2}\left[4\left(\bar{g}_{\infty}-\omega\right)^{2}-W^{2}-4\right]}\right) \frac{1}{K}
$$

This result is depicted in Fig. 8 for three different values of the branching parameter: $K=1,2$, and 3. Qualitatively, these curves look the same. Naturally the correction becomes stronger for decreasing $K$. At the band edges the DOS diverges and becomes negative (not indicated in the figure). As mentioned, this problem is related to the fact that only the corrections of order $K^{-1}$ are taken into account. To resolve the problem, one has to include higher orders in $1 / K$ in a suitable manner, e.g., self-consistently in the parameter $1 / K$.

At the band center a lowering of the DOS is observed, and at the sides $(\omega \approx \pm 2.9)$ two peaks appear. These peaks are also known to be present in the free-electron limit for $K \geq 2$. Qualitatively, the result depends only weakly on the strength of the disorder. For decreasing disorder the distance between the two minima decreases, until they merge for some finite value of the disorder.

\section{B. Binary alloy}

The binary-alloy model is the most extensively studied case, especially within the CPA. ${ }^{9,10}$ Solving Eqs. (2) and

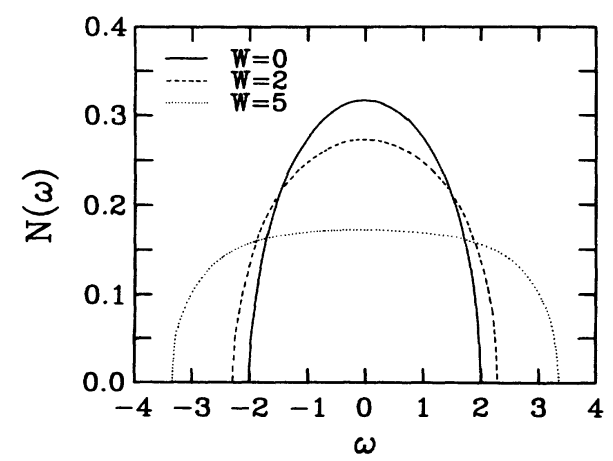

FIG. 7. DOS for the box disorder distribution on a Bethe lattice with infinite branching for three different disorder strengths $(\boldsymbol{W})$.
(28) for the binary-alloy distribution, Eq. (5), a cubic equation for $\bar{g}_{\infty}$ is obtained,

$$
\begin{aligned}
& \bar{g}_{\infty}^{3}(\omega)-(2 \omega) \bar{g}_{\infty}^{2}(\omega)+\left(\omega^{2}-W^{2} / 4+1\right) \bar{g}_{\infty}(\omega) \\
&-[\omega+(2 c-1) W / 2]=0 .
\end{aligned}
$$

The DOS is plotted in Fig. 9 for different values of the disorder strength and relative weight $c$ (concentration of impurities) of the $\delta$-functions. We see that a band gap opens for a finite value of the disorder. Our results may be compared with those by Velický et al. ${ }^{8}$ These authors applied the CPA in combination with the semielliptical model DOS, which we identify to be exact on the Bethe lattice with infinite branching.

Calculation of the $1 / K$ corrections using Eq. (30) is easy because of the simple structure of Eq. (5). In Fig. 9 the result for the values $K=2$ and 3 is shown in comparison with the result for infinite branching. As in the case

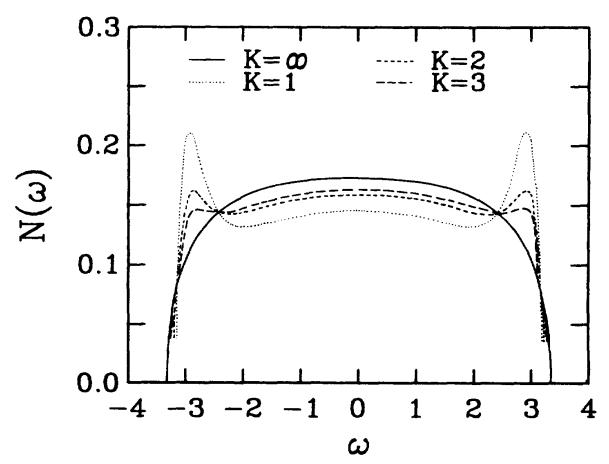

FIG. 8. DOS for the box disorder distribution on a Bethe lattice with infinite branching, compared with calculations including corrections of order $1 / K$ for various values of $K ; W=5$ in all cases. 

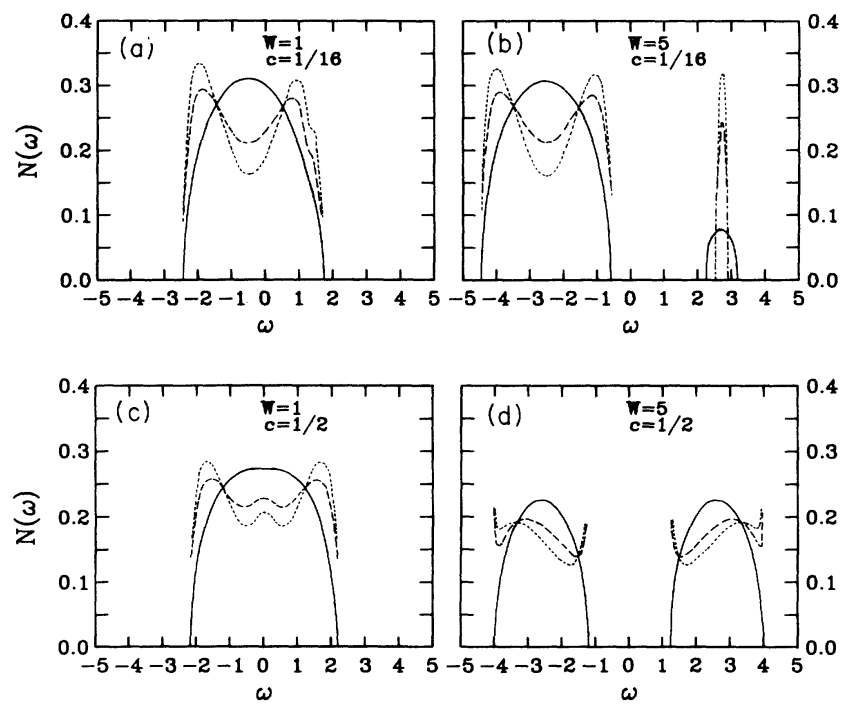

FIG. 9. DOS for the binary-alloy model on a Bethe lattice with infinite branching, compared with calculations including corrections of order $1 / K$ for various values of $K$ : solid curve, $K=\infty$; long-dashed curve, $K=3$; short-dashed curve, $K=2$. (a) $W=1, c=1 / 16$. (b) $W=5, c=1 / 16$. (c) $W=1, c=1 / 2$. (d) $W=5, c=1 / 2$.

of the "box" distribution, a more pronounced structure emerges and the DOS diverges at the band edges, which signals the breakdown of the validity of the $1 / K$ expansion as discussed in Sec. V A. In Figs. 9(a) and 9(b) the DOS is shown for $c=1 / 16$, where we can distinguish a host and an impurity band. In the host band the DOS is lowered and the two peaks, known to be present also in the free-electron limit, appear. In the impurity band, however, we observe an increase of the DOS. In the split-band situation these effects appear isolated [Fig. $9(\mathrm{~b})$ ], whereas in the single-band situation it results in a kink in the DOS [Fig. 9(a)]. For $c=1 / 2$, Figs. 9(c) and 9(d), similar behavior is observed. In the split-band case the (unphysical) divergence at the band edges is particularly large and dominates the DOS-enhancement peaks for low $|\omega|$. In the single-band case these peaks give rise to an increase of the DOS at $\omega=0$, in addition to the overall decrease of the DOS at the center.

\section{Hypercubic lattices}

For a Bethe lattice, the DOS, Eq. (39), is seen to be bounded for all $Z$, and corrections to the limit $Z \rightarrow \infty$ are analytic in $1 / Z$. In contrast, for a hypercubic lattice the DOS acquires infinite (exponential) tails in the limit $Z \rightarrow \infty$, and for finite $Z$ there are not only corrections of order $Z^{-n}$, but also nonanalytic corrections of order $\exp (-Z)$, due to van Hove singularities. ${ }^{27,28}$ The latter corrections cannot be treated in perturbation theory in $1 / Z$. To work on a hypercubic lattice for finite $d=Z / 2$, starting from $d=\infty$, we must therefore use the exact $d$ dimensional DOS for free lattice electrons. If on the diagrammatic level we include corrections of order $d^{-n}$, the error introduced thereby will clearly be one order higher, i.e., of order $d^{-(n+1)}$, apart from nonanalytically small corrections.

In particular, inserting the exact, free $d$-dimensional DOS into the results for $d \rightarrow \infty$, the CPA for a $d$ dimensional system is recovered exactly. In the propagator formalism, such a procedure means that we work with the exact free propagator for $d$ dimensions instead of the one for $d \rightarrow \infty$. On the other hand, in the locator formalism this implies that the index relaxation is not applied in the simplification scheme discussed in Sec. III. Using this technique in combination with perturbation theory in $1 / d$, one obtains the $1 / d$ expansion around the CPA.

\section{DISCUSSION}

We have shown that the limit of large coordination numbers allows for the formulation of a self-consistent, conserving (see Appendix B), mean-field theory for oneparticle properties in the disordered-electron problem. We derived the CPA within the RPE locator formalism, and proved that this approximation becomes exact in the limit $Z \rightarrow \infty$, irrespective of lattice type. As a consequence, the quantitative and qualitative success of the CPA even for intermediate values of the disorder and the impurity concentration may easily be understood. The astonishing fact that the CPA can be formulated in terms of diagrams that are structurally equivalent in both the locator and propagator formalisms also becomes clear. The reason for this lies in the equivalence of the scaling of the hopping integral and the bare propagator, respectively.

Owing to the existence of a small parameter, $Z^{-1}$, which is independent of the original input parameters of the model (disorder, impurity concentration), corrections to the mean-field theory can be calculated systematically within perturbation theory in $Z^{-1}$. For this it is essential that the correct scaling of the hopping integral, with $1 / \sqrt{Z}$, is used. Therefore the above mean-field theory is a controlled approximation.

Our theory does not yet include the $1 / Z$ corrections on a self-consistent level. Hence our present results cannot describe the behavior close to the band edges, where pure perturbation theory is bound to fail. We expect that, in contrast to previous attempts (for a discussion, see Ref. 23), a self-consistent $1 / Z$ expansion can be constructed that has the required analytical properties, ${ }^{29}$ as is the case for the CPA. ${ }^{30}$ An alternative approach, for which selfconsistency is not required, is the traveling-cluster approximation introduced by Mills and Ratanavararaksa. ${ }^{31}$

The applications discussed in this paper (i.e., disordered systems on a Bethe lattice with box- or binary-alloy disorder distributions) show that results can be obtained in a straightforward manner. For the box model a band broadening is found, in agreement with earlier results. ${ }^{26}$ An explicit expression for the bandwidth was derived. By including $1 / K$ corrections, some of the structure known to exist in the free-electron case for $K \geq 2$ is retained. In the binary-alloy model we rederived a result of Velický et $a l .^{8}$ In their paper the equation for the DOS 
was a result of using the CPA in combination with the semielliptical model DOS for the free-electron problem. In our formulation this approximation is shown to become exact for disordered electrons on a Bethe lattice.

While the limit $Z \rightarrow \infty$, and hence the CPA, has proved to be a very useful approximation scheme for the calculation of one-particle properties, it is inadequate to describe two-particle properties, e.g., the dynamical conductivity. It is well known that, for finite disorder, localized states cannot exist in any single-site theory such as the CPA. ${ }^{32}$ This is also clear from a diagrammatic point of view, since in the limit $Z \rightarrow \infty$ the retarded and advanced Green functions entering into two-particle quantities essentially decouple, leaving no room for the important quantum-mechanical multiple-scattering processes that are essential for the Anderson transition. ${ }^{33}$ Simple perturbation theory in $1 / Z$ does not alter this picture; the essential point is that the $1 / Z$ corrections again have to be incorporated self-consistently to yield a localization transition. The construction of such a theory is presently under investigation.

\section{ACKNOWLEDGMENTS}

We would like to thank Dr. V. Janiš, Dr. W. Metzner, Dr. F. Gebhard, and Professor E. Müller-Hartmann for valuable discussions. This work was supported, in part, by Sonderforschungsbereich No. 341 of the Deutsche Forschungsgemeinschaft.

\section{APPENDIX A: 1 /K CORRECTIONS FOR THE BETHE LATTICE}

The $1 / K$ correction, Eq. (30), will now be derived. The general form of the averaged full locator [Eq. (12)] is given by

$$
\left\langle\left\langle g_{i}\right\rangle\right\rangle=\left\langle\left\langle\sum_{n=0}^{\infty}\left(g_{i}^{0}\right)^{n+1}\left\langle\left\langle\left(\sigma_{i}\right)^{n}\right\rangle\right\rangle\right\rangle .\right.
$$

The averaging over the different self-energies does not decouple for $K<\infty$. Instead, we have to write

$$
\begin{aligned}
& \left\langle\left\langle\left(\sigma_{i}\right)\right\rangle \geq\left\langle\left\langle\sigma_{i}\right\rangle\right\rangle^{n}+\frac{n(n-1)}{2}\left\langle\left\langle\sigma_{i}\right\rangle\right\rangle^{n-2} \overline{\sigma \sigma},\right. \\
& \overline{\sigma \sigma} \equiv \sum_{j}\left(\left\langle\left\langle\sigma_{i,(j)} \sigma_{i,(j)}\right\rangle\right\rangle-\left\langle\left\langle\sigma_{i,(j)}\right\rangle\right\rangle\left\langle\left\langle\sigma_{i,(j)}\right\rangle\right\rangle\right) .
\end{aligned}
$$

The first term in the above expansion, Eq. (A2), results from the limit $K \rightarrow \infty$; the second is the first correction. The subscript $i,(j)$ indicates that the two hopping matrix elements occurring in the definition of the self-energy, Eq. (27), are connected to the site $j$, i.e., $\sigma_{i,(j)}=t_{i j} g_{j}^{-(i)} t_{j i}$. Substitution of Eq. (A2) into Eq. (A1), and carrying through the different sums, results in

$$
\bar{g}=\left\langle\left\langle\frac{g_{i}^{0}}{1-g_{i}^{0} \bar{\sigma}}\right\rangle\right)+\overline{\sigma \sigma}\left\langle\left\langle\frac{\left(g_{i}^{0}\right)^{3}}{\left(1-g_{i}^{0} \bar{\sigma}\right)^{3}}\right\rangle\right\rangle .
$$

Let us first have a look at the term $\overline{\sigma \sigma}$, which is already of order $1 / K$ and thus allows for the replacement of $\bar{\sigma}$ by $\bar{\sigma}_{\infty}$ in the second term of Eq. (A4). Using the relation for the self-energy on the Bethe lattice [Eq. (27)], the first term of $\overline{\sigma \sigma}$ is given by

$$
\begin{aligned}
\left\langle\left\langle\sigma_{i,(j)} \sigma_{i(j)}\right\rangle\right\rangle & =t_{i j}^{2}\left\langle\left\langle g_{j}^{-(i)} g_{j}^{-(i)}\right\rangle\right\rangle t_{i j}^{2} \\
& =t^{4}\left\langle\left\langle\frac{\left(g_{i}^{0}\right)^{2}}{\left(1-g_{i}^{0} \bar{\sigma}_{\infty}\right)^{2}}\right\rangle\right\rangle .
\end{aligned}
$$

In Eq. (A5) we expanded both locators into a geometric series as in Eq. (A1), and resummed the result. Since $\overline{\sigma \sigma}$ is already of order $1 / K$, we were allowed to substitute $\bar{\sigma}_{\infty}$ for the emerging $\left\langle\left\langle\sigma_{j}^{-(i)}\right\rangle\right\rangle$. The second term of $\overline{\sigma \sigma}$ simply gives $\left\langle\left\langle\sigma_{i,(j)}\right\rangle\right\rangle\left\langle\left\langle\sigma_{i,(j)}\right\rangle\right\rangle=t^{2} \bar{g}^{[1]} t^{2} \bar{g}^{[1]}$, in which $\bar{g}^{[1]}$ may also be replaced by $\bar{g}_{\infty}$. Thus we obtain

$$
\overline{\sigma \sigma}=(K+1) t^{4}\left(\left\langle\left(\frac{\left(g_{i}^{0}\right)^{2}}{\left(1-g_{i}^{0} \bar{\sigma}_{\infty}\right)^{2}}\right\rangle\right)-\bar{g}_{\infty}^{2}\right) .
$$

For $\bar{\sigma}$, appearing in the factor $\left\langle\left\langle\sigma_{i}\right\rangle\right\rangle^{n-2}$ from Eq. (A2), we write

$\bar{\sigma}=(K+1) t^{2} \bar{g}^{[1]}$,

and, for $\bar{g}^{[1]}$, a relation analogous to Eq. (A4) is deduced:

$\bar{g}^{[1]}=\left\langle\left\langle\frac{g_{i}^{0}}{1-g_{i}^{0} \bar{\sigma}^{[1]}}\right\rangle+\overline{\sigma \sigma}{ }^{[1]}\left\langle\left\langle\frac{\left(g_{i}^{0}\right)^{3}}{\left(1-g_{i}^{0} \bar{\sigma}_{\infty}\right)^{3}}\right\rangle\right.\right.$,

$\overline{\sigma \sigma}^{[1]} \equiv \sum_{k}\left(\left\langle\left\langle\sigma_{j,(k)}^{-(i)} \sigma_{j,(k)}^{-(i)}\right\rangle\right\rangle-\left\langle\left\langle\sigma_{j,(k)}^{-(i)}\right\rangle\right\rangle\left\langle\left\langle\sigma_{j,(k)}^{-(i)}\right\rangle\right)\right.$.

Thereby two new terms are generated, $\bar{\sigma}^{[1]}$ and $\overline{\sigma \sigma}^{[1]}$. However, since we are only interested in corrections up to $1 / K$, these are given by

$$
\bar{\sigma}^{[1]}=K t^{2} \bar{g}^{[1]}, \quad \overline{\sigma \sigma}^{[1]}=\overline{\sigma \sigma} .
$$

This closes the set of equations. Note that in Eq. (A10) one cannot replace $\bar{g}^{[1]}$ by $\bar{g}_{\infty}$ because this would violate the self-consistency.

By setting $\bar{g}^{[1]} \equiv \bar{g}_{\infty}+\bar{g}_{1 / K}^{[1]}$, the system of Eqs. (A4), (A7), (A8), and (A10) can be solved for $\bar{g}_{1 / K}^{[1]}$, yielding

$$
\bar{g}_{1 / K}^{[1]}=\frac{\overline{\sigma \sigma}\left\langle\left\langle\left(g_{i}^{0}\right)^{3} /\left(1-g_{i}^{0} \bar{\sigma}_{\infty}\right)^{3}\right\rangle\right\rangle}{1-K t^{2}\left\langle\left(\frac{\left(g_{i}^{0}\right)^{2}}{\left(1-g_{i}^{0} \bar{\sigma}_{\infty}\right)^{2}}\right\rangle\right)} .
$$

Thus $\bar{g}^{[1]}$ is known, whereby $\bar{\sigma}^{[1]}$ and $\bar{\sigma}$ are determined by Eqs. (A10) and (A7). This is used in Eqs. (A6) and (A4) to find $\bar{g}$. The result is given in Eq. (30).

\section{APPENDIX B: EXACTNESS OF THE CPA FOR $Z \rightarrow \infty$ (PROPAGATOR PROOF)}

In the propagator formalism the mathematical formulation of the CPA is well known and has a simple structure ${ }^{4,5,9,10}$ see Eq. (31). The exactness of the CPA in the limit $Z \rightarrow \infty$ can also be proved within this formalism. However, for the proof to be lattice independent, one cannot employ a momentum representation (on the Bethe lattice, $\mathbf{k}$ states are not defined). In contrast to the locator formalism, the bare Green function in the propagator formalism (bare propagator), $G_{i j}^{0}(\omega)$, depends on $Z$; in particular, for large $Z,{ }^{13,28}$ 


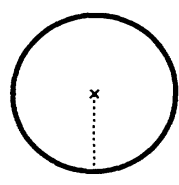

(a)

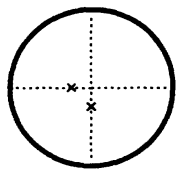

(d)

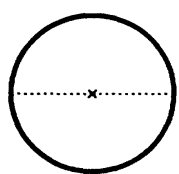

(b)

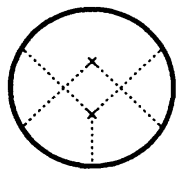

(e)

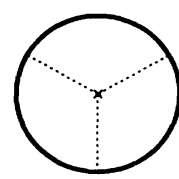

(c)

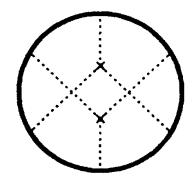

$(t)$
FIG. 10. Different diagrammatic contributions to the generating functional $\Phi$. (a) Single, (b) double, and (c) triple scattering at a single impurity. (d)-(f) Several pair contributions to $\Phi$.

$$
G_{i j}^{0} \propto Z^{-|i-j| / 2},
$$

such that ${ }^{34}$

$$
\sum_{n} G_{i n}^{0} \varepsilon_{n} G_{n j}^{0} \propto Z^{-|i-j| / 2} .
$$

To do power counting in $1 / Z$ for one-particle quantities, the generating-functional approach of Baym and $\mathrm{Ka}$ danoff ${ }^{35,36}$ can be used. The self-energy, $\bar{\Sigma}_{i j}(\omega)$, is deduced from some functional $\Phi$ by $\bar{\Sigma}_{i j}(\omega)=\delta \Phi / \delta \bar{G}_{i j}(\omega)$. In general, $\Phi$ is given by all irreducible diagrams equipped with the proper weight factor, depending on its symmetry. Some diagrams are given in Fig. 10. Note that the cross indicates a cumulant $C(l)$ (instead of the impurities itself), where $l$ is the number of scattering processes at the impurity, so that the MOC is taken into account automatically. There is a simple way to calculate this generating function for $C(l)$ (see Ref. 15), and it is given by $f(x)=\ln \langle\langle\exp (x \varepsilon)\rangle$.
Taking the limit $Z \rightarrow \infty$, we find that all diagrams with two or more nondiagonal propagators disappear. For example, in Fig. 10, diagrams (a) - (c) are of order 1 because they consist only of diagonal propagators. Figures 10(d) and $10(e)$ are at least of order $Z^{-1}$, as a result of four offdiagonal propagators and one free site. Finally, Fig. 10(f) is at least of order $Z^{-2}$. Therefore, we end up with a series of diagrams such as Figs. $10(a)-10(c)$. Hence, the self-energy becomes diagonal. On the other hand, the severe reduction of the relevant diagrams implies that all MOC diagrams that result from diagrams that we eliminated in the limit $Z \rightarrow \infty$ must also be left out. A resummation of the remaining diagrams is possible within the diagrammatic approach of Leath. ${ }^{17}$ Following his method for the calculation of the modified cumulants, $C_{\infty}(l)$, one finds, using a generating function $g(x)$ defined as

$$
g(x) \equiv \sum_{l=1}^{\infty} C_{\infty}(l) x^{l-1},
$$

and using a similar definition for the generating function for the disorder distribution, $D(x)$,

$$
D(x) \equiv \sum_{l=1}^{\infty}\left\langle\left\langle\varepsilon^{l}\right\rangle\right\rangle x^{l-1},
$$

that $g(x)$ and $D(x)$ satisfy

$$
\begin{aligned}
& g(y)=\frac{D(x)}{1+x D(x)}, \\
& y=\frac{x}{1-x g(y)} .
\end{aligned}
$$

Since $\bar{\Sigma}_{i i}=g\left(\bar{G}_{i i}\right)$, one recovers, after some algebraic manipulations, the CPA formula [Eq. (31)] . From this approach it is also immediately clear that our mean-field theory (and CPA) is conserving in the sense of Baym and Kadanoff. $^{35,36}$
${ }^{1}$ P. Lloyd, J. Phys. C 2, 1717 (1969).

${ }^{2}$ F. Wegner, Z. Phys. B 51, 279 (1983).

${ }^{3}$ P. Erdös and R. C. Herndon, Adv. Phys. 31, 65 (1982).

${ }^{4}$ J. M. Ziman, Models of Disorder (Cambridge University Press, Cambridge, UK, 1979).

${ }^{5}$ E. N. Economou, Green's Functions in Quantum Physics (Springer, Berlin, 1979).

${ }^{6}$ P. Soven, Phys. Rev. 156, 809 (1967).

${ }^{7}$ D. W. Taylor, Phys. Rev. 156, 1017 (1967).

${ }^{8}$ B. Velický, S. Kirkpatrick, and H. Ehrenreich, Phys. Rev. 175, 747 (1968).

${ }^{9}$ R. J. Elliott, J. A. Krumhansl, and P. L. Leath, Rev. Mod. Phys. 46, 465(1974).

${ }^{10}$ F. Yonezawa and K. Morigaki, Suppl. Prog. Theor. Phys. 53, 1 (1973).

${ }^{11}$ R. Brout, Phys. Rev. 118, 1009 (1960).

${ }^{12}$ P. A. Pearce and C. J. Thompson, Commun. Math. Phys. 58, 131 (1978).
${ }^{13}$ W. Metzner and D. Vollhardt, Phys. Rev. Lett. 62, 324 (1989).

${ }^{14}$ For reviews, see D. Vollhardt, Physica B 169, 277 (1991); E. Müller-Hartmann, Int. J. Mod. Phys. B 3, 2169 (1989).

${ }^{15}$ L. Schwartz and E. Siggia, Phys. Rev. B 5, 383 (1972).

${ }^{16}$ P. W. Anderson, Phys. Rev. 109, 1492 (1958).

${ }^{17}$ P. L. Leath, Phys. Rev. B 2, 3078 (1970).

${ }^{18}$ R. Abou-Chacra, P. W. Anderson, and D. J. Thouless, J. Phys. C 6, 1734 (1973).

${ }^{19}$ P. L. Leath, J. Phys. C 6, 1559 (1973).

${ }^{20}$ E. Müller-Hartmann (unpublished).

${ }^{21}$ G. Czycholl (private communication).

${ }^{22}$ B. G. Nickel and J. A. Krumhansl, Phys. Rev. B 4, 4354 (1971).

${ }^{23}$ G. Czycholl and J. Zittartz, Z. Phys. B 30, 375 (1978).

${ }^{24}$ C. T. White and E. N. Economou, Phys. Rev. B 8, 3742 (1977).

${ }^{25}$ J. Hubbard, Proc. R. Soc. London, Ser. A 276, 238 (1963); 281, 401 (1964).

${ }^{26}$ F. Brouers, J. Phys. C 4, 773 (1971). 
${ }^{27}$ U. Wolff, Nucl. Phys. B225 [FS9], 391 (1983).

${ }^{28}$ E. Müller-Hartmann, Z. Phys. B 74, 507 (1989).

${ }^{29}$ At least for the Bethe lattice, we have been able to derivefor arbitrary $Z$-an exact integral equation in closed form for the $n$-particle Green function, which indicates that a selfconsistent $1 / Z$ expansion with the correct analytical properties exists.

${ }^{30}$ E. Müller-Hartmann, Solid State Commun. 12, 1269 (1973).

${ }^{31}$ R. Mills and P. Ratanavararaksa, Phys. Rev. B 18, 5291
(1978).

${ }^{32}$ R. Haydock and A. Mookerjee, J. Phys. C 7, 3001 (1974).

${ }^{33}$ E. Abrahams, P. W. Anderson, D. C. Licciardello, and T. V. Ramakrishnan, Phys. Rev. Lett. 42, 673 (1979).

${ }^{34}$ P. G. J. van Dongen, F. Gebhard, and D. Vollhardt, Z. Phys. B 76, 199 (1989).

${ }^{35}$ G. Baym and L. P. Kadanoff, Phys. Rev. 124, 287 (1961).

${ }^{36}$ G. Baym, Phys. Rev. 127, 1391 (1962). 